\title{
Stress Analysis of Conical Contact Joints in the NIST 4.45 MN Deadweight Machine
}

\author{
Nicholas Vlajic, Akobuije Chijioke, and Rick Seifarth \\ National Institute of Standards and Technology, \\ Gaithersburg, MD 20899
}

nicholas.vlajic@nist.gov

ako.chijioke@nist.gov

ricky.seifarth@nist.gov

During the disassembly phase of the refurbishment of the National Institute of Standards and Technology 4.45 MN Deadweight Machine, workers confirmed previously suspected damage in certain conical contact joints within the apparatus. A finite element analysis of the contact joints is performed with the aim of understanding some of the potential origins of failure in order to prevent future damage in the machine. Parametric studies are performed using a finite element model while varying geometry, material constitutive relations, and frictional forces at the interface of the convex and concave cones. The numerical solutions treat nonlinearities originating from elastic-plastic material relations, frictional forces, and contact between the surfaces. These results provide insights into the mechanisms of stress generation in such joints, and how these stresses are influenced by different structural parameters.

Key words: deadweight machine; galling; nonlinear finite elements; seizure.

Accepted: April 25, 2016

Published: May 10, 2016

http://dx.doi.org/10.6028/jres.121.010

\section{Introduction}

The National Institute of Standards and Technology (NIST) Mass and Force Group provides SItraceable force calibration services ranging from $44 \mathrm{~N}$ to $4.448 \mathrm{MN}$ based on primary deadweight force standards using a series of six deadweight force machines (DWMs) [1]. Typically, these deadweight machines are used to calibrate elastic force transducers (load cells), which output a voltage when a force is applied to the transducer. These six machines have maximum force capacities of $2.25 \mathrm{kN}(0.505 \mathrm{klbf})$, $27.1 \mathrm{kN}$ (6.1 klbf), $113 \mathrm{kN}$ (25.3 klbf), $498 \mathrm{kN}$ (112 klbf), 1.33 MN (300 klbf), and $4.45 \mathrm{MN}$ (1 $000 \mathrm{klbf})$, and are housed in a special building with strictly controlled environmental conditions located on the NIST Gaithersburg, MD campus. In these machines, the force applied to a transducer is generated by Earth's gravity acting on a calibrated mass, which may be written $F=m g$ where $m$ is mass and $g$ is Earth's gravitational acceleration, wherein the mass has been corrected for buoyancy effects of the local atmosphere. In general, the calibration uncertainty achieved using these machines is transducer-dependent, but expanded uncertainties $(k=2)$ can be as small as $0.0012 \%$ [2].

The largest DWM is the $4.45 \mathrm{MN}$ apparatus, which has a height of over $25 \mathrm{~m}$ and has been in use in the NIST Force Laboratory since 1965. Starting from its first years of operation, this machine has been used to provide force calibrations to industry, government labs, and academia for numerous applications including for example space programs. This DWM is presently the largest deadweight force standard in the world, and has been used to perform thousands of force transducer calibrations to date, with each calibration typically involving several applications of the full 4.45 MN force. It was the centerpiece of two International Key Comparisons of primary large force measurements at several national metrology 
institutes across the world; thus, the 4.45 MN DWM is an international resource for SI-traceable measurements of large forces which has disseminated force units to a worldwide customer base.

\subsection{Operation and History of the Deadweight Machines}

Calibrations of force transducers are performed by applying a discrete set of applied forces to the transducer by the addition of individual stainless steel weights ${ }^{1}$ to the supported load. There are two different operational designs of DWMs at NIST [3]. In the three smaller-capacity DWMs, the transducer is loaded by a main shaft, and individual weights, which are otherwise held up by hydraulic or pneumatic actuators, are lowered onto the shaft. In this design the operator can choose which weights to load onto the shaft and consequently, the transducer. Measurements may thus be made for a large number of different force values, as the various possible combinations of weights provide a large number of available mass values.

The design and operation of the three larger capacity DWMs is illustrated in Fig. 1, which specifically depicts the 4.45 MN DWM. In this design, a force transducer is placed between a lifting frame and a yoke the yoke itself is weight No. 1. A hydraulic lift then picks up the loading frame, transducer, and yoke assembly to load the transducer. As the assembly is raised, each weight is picked up in turn by the weight above it until the transducer is loaded with the desired force. This weight stack design is described as chainloading or serial-loading, meaning that each weight in the stack must support all weights below it when forces are generated during machine use. The physical connection between the weights is made between the bottom plate of the top weight body and the pick-up stud of the weight body directly below, as shown in Fig. 1. This interface is a conical contact joint. As larger forces are generated by the addition of weights to the hanging stack, the forces passing through the pick-up stud/center hub interfaces of the weights in the upper third of the weight stack produce the highest stresses. Sufficiently high stresses at conically seated interface surfaces can lead to plastic deformation. Both the 1.33 MN and the $498 \mathrm{kN}$ DWMs were disassembled in 1975 and 1988, respectively, in order to repair damage done on the conical joints between the pick-up studs and the bottom hub plates. Although the forces realized in these machines are smaller than the forces realized in the 4.45 MN DWM, the contact area of the joints is much smaller, so the stresses may still be large. The damaged parts cannot be visually observed without disassembling the machine, and the damage was inferred from the characteristic behavior of the machine when unloading the masses. In both instances, the damage to the surfaces was identified to be galling damage (discussed below). In each machine the galled surfaces were re-machined and treated with a solid lubricant.

\subsection{Damage in the 4.45 MN Machine}

In recent years, the 4.45 MN DWM started to show similar indications of damage as had earlier been observed in the two other DWMs of similar chain-loading design. From previous operator experience with the other DWMs, the telltale indication of damage at the contact joints is an impulse generated in the machine while unloading the weight stack, caused by the surfaces of pick-up stud and bottom hub plate, fusing together during loading and subsequently breaking free during unloading. The suspected damage worsened over time and the decision to restore the machine in order to prevent further deterioration was made in 2013. In 2014, the first ten masses were disassembled and removed from the machine. As had been suspected, damage was observed on the bottom hub plate of weights No. 3 and No. 4 and the pick-up studs of weights No. 4 and No. 5. Photographs of the damage on the bottom hub plate and pick-up stud are provided in Fig. 2. It is interesting to note that the first pick-up stud (in weight No. 3) and the lower nut of the yoke (see Fig. 1) showed no observable sign of surface damage.

\footnotetext{
${ }^{1}$ Within this document weight refers to the physical object, namely the stainless steel body, that is used to realize force; it is not to be mistaken for a quantity with associated units, such as force or mass.
} 

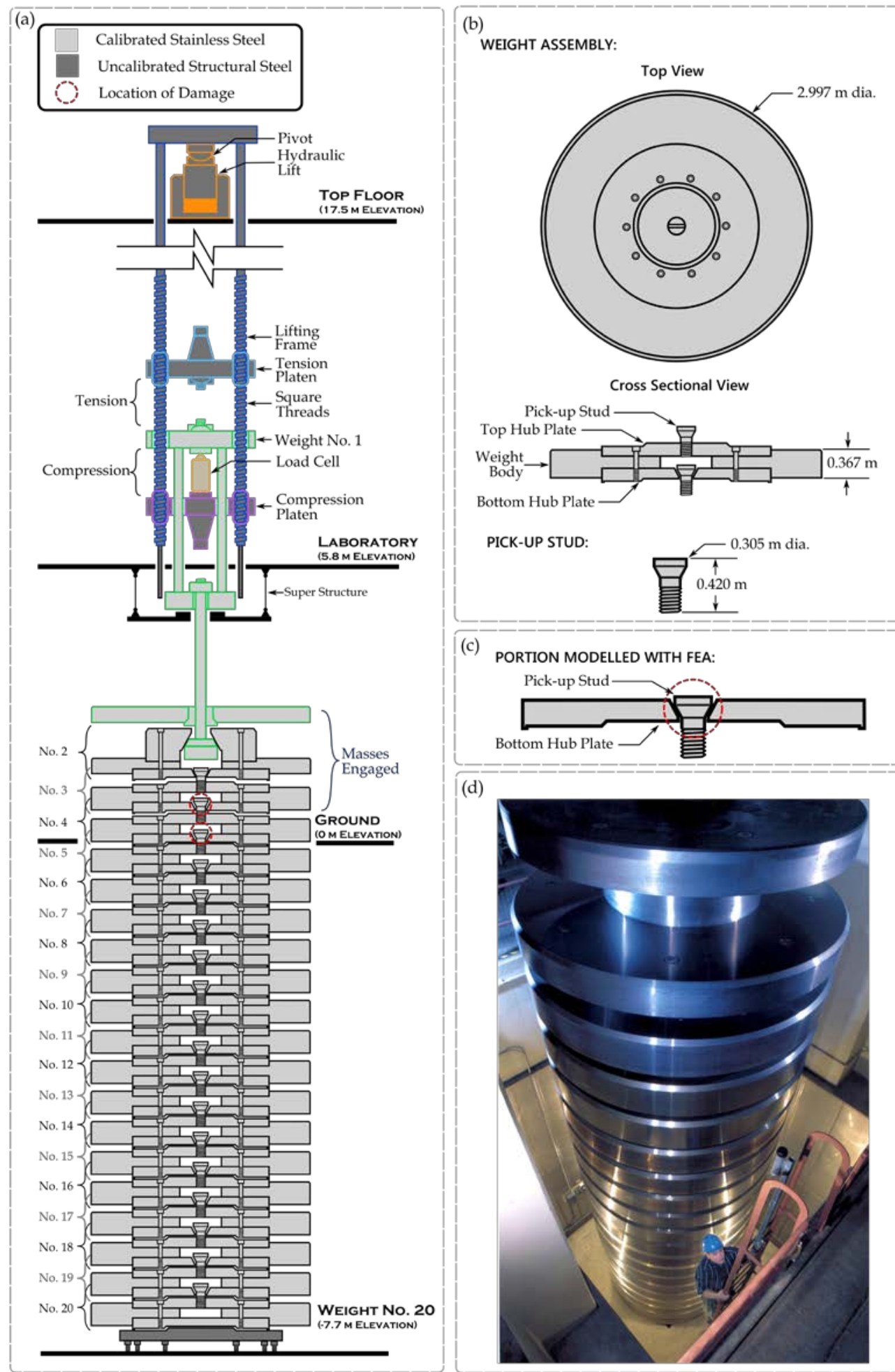

(c) PORTION MODELLED WITH FEA:
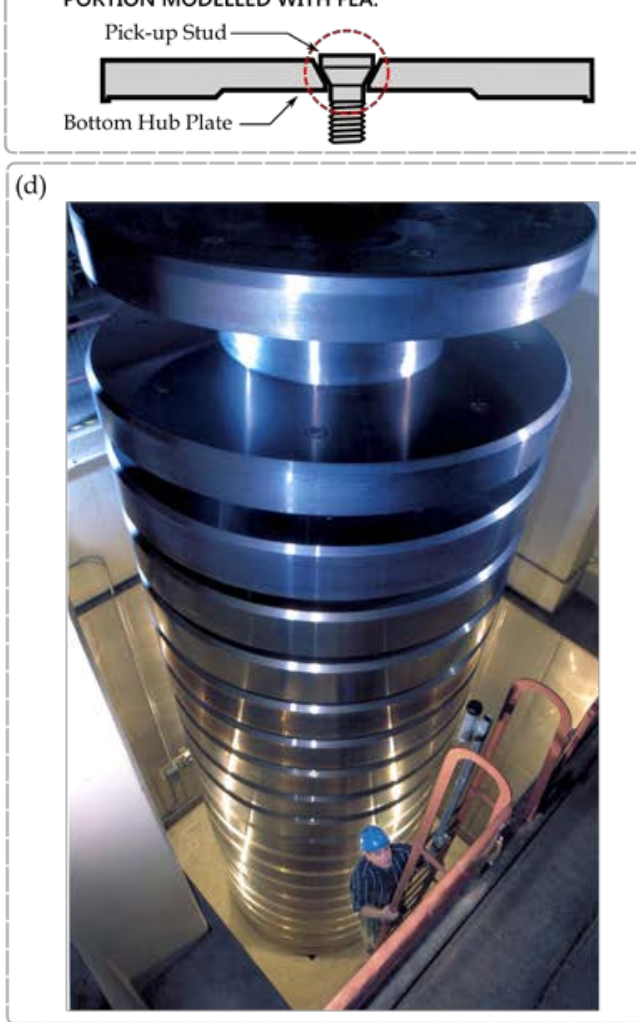

Fig. 1. (a) A schematic of the NIST 4.45 MN machine, where the two damaged studs and hubs are highlighted in dashed, red circles, (b) detailed view of one of the weight assemblies, (c) the portion of the machine modelled with finite elements, and (d) a photograph of the weight stack. Each assembled weight generates a force of $222.4 \mathrm{kN}$ (roughly the weight of ten minivans) when suspended in a gravitational field. 

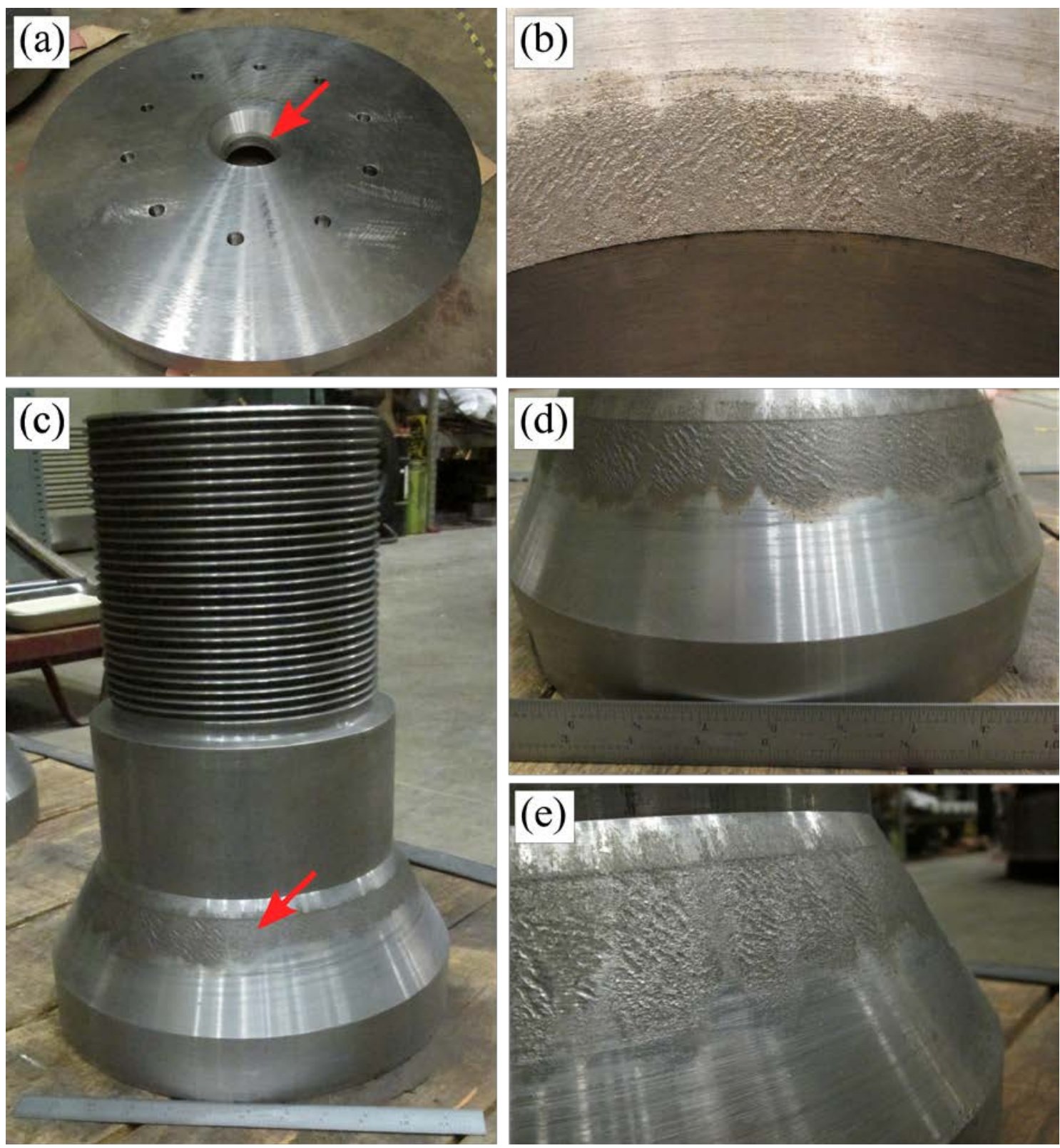

Fig. 2. Photographs of components of the machine that exhibit surface damage: (a) bottom hub plate (outer diameter of $1.829 \mathrm{~m}$ ), (b) close-up of bottom hub plate, (c) pick-up stud (neck diameter of $0.2159 \mathrm{~m}$ ), (d) close-up of galling damage on pick-up stud, and (e) additional close-up of galling damage on the pick-up stud. It is noted that the stud shown in figures (c), (d), and (e) is up-side-down from its orientation in the machine. 


\subsection{Accuracy of the 4.45 MN Machine}

A number of indicators show that no measurements made with this machine have had their accuracy affected by the observed damage. The damaged weights are high in the stack-assembly and any potential errors would be most prominent at the $667 \mathrm{kN}, 890 \mathrm{kN}$, and the $1.11 \mathrm{MN}$ force measurement points. Inhouse NIST intercomparisons between these measurement points and the corresponding measurement points in the 1.33 MN DWM showed good agreement, which indicates that the damage has not affected the accuracy of the machine. Moreover, repeated tracking measurements performed with different load cells and transducers over a fifty-year history suggest no change in the forces generated by the machine.

In addition to the in-house intercomparisons and machine history, two international intercomparisons also reinforce confidence in the integrity of the forces realized in the 4.45 MN DWM. In 1989, an intercomparison between NIST and Physikalisch-Technische Bundesanstalt (PTB), Germany demonstrated agreement to within $\pm 40 \mathrm{ppm}$ for forces up to $900 \mathrm{kN}$ [4]. Furthermore, an international force key comparison performed in this machine as part of the US commitment to the Comité International des Poids et Mesures (CIPM)/Bureau International des Poids et Mesures (BIPM) arrangement to promote uniformity and agreement of measurements among National Metrology Institutes (NMIs), also yielded successful measurement results [5].

\subsection{Damage Mechanisms}

At this point we discuss potential damage mechanisms in relation to the damage observed on the 4.45 MN DWM. Galling is a form of adhesive wear that results in macroscopic particles of material being torn (and possibly transferred) between two surfaces, constituting large and irreversible damage. Galling originates when two surfaces rub together and asperities are detached from the surface, creating small wear particles, which eventually lead to tearing of larger pieces of material [6]. Additionally, these joints are likely susceptible to material seizure. Seizure, also referred to as cold welding, is also form of adhesive wear, where two surfaces that are free of sufficient oxide and film, come into contact and form a bond through molecular (atomic) interactions [7]. A contact joint is more susceptible to seizure when the two surfaces are of the same material, and material may be transferred from one surface to another [7].

In addition to adhesive wear, the conical-joint seats are also subject to loading and unloading when picking up weights. This loading and unloading can result in shakedown. Shakedown is not a form of wear, but a process that results in work hardening described as follows. When surfaces are loaded to the point of plastic deformation and subsequently unloaded, a residual stress is left in the surface resulting in strain hardening. After many such loading cycles, the surface may be able to hold the entire load elastically. This process is referred to as elastic shakedown, and is generally considered to be a beneficial effect. However, if the loads are high enough in magnitude and the surface material can be described by a nonlinear strain hardening model (typical for many alloys), the surfaces may start to undergo plastic ratcheting (or cyclic creep), wherein gross deformations of the surface may accumulate [6]. A detailed study of shakedown and ratcheting is provided in Refs. [8, 9] and the works cited therein. In this paper, we refer to galling as the type or form of material damage, although we acknowledge that there may be other types of wear and wear processes in addition to those discussed above (e.g., fretting) that may be relevant to the investigated scenario. Although galling can lead to material transfer, this did not occur to a level that affected any calibration results, as described above.

\subsection{Goal and Organization}

In this paper, we model the conical contact joints of the 4.45 MN DWM and perform parametric studies in order to gain some understanding of the origin of the damage observed, and of potential means of preventing similar damage from reoccurring in the future. The remaining sections of this paper are as follows. In Sec. 2, a finite element model of the top hub plate and pick-up stud is described, and studies of the boundary conditions, material constitutive relations, and analysis of errors are presented. In Sec. 3, results of parametric studies of the effects of the dimensional tolerances and interface friction are presented. In Sec. 4, conclusions from this study are summarized. 


\section{Structural Modelling}

We now describe structural finite element modelling of the components of the 4.45 MN DWM, which was performed using the software package COMSOL Multiphysics $4.4^{2}$. In considering the potential sources of uncertainty in modelling the components of the 4.45 MN DWM (e.g., friction, boundary conditions, material constitutive relations), we perform parameter sweeps for a given unknown or model two opposing extrema between which the true solution lies. Friction and geometric tolerances are studied via parametric sweeps, while two different material constitutive relations are implemented (linear-elastic and linear-elastic, perfectly plastic). Lastly, two different sets of boundary conditions are considered.

The solutions determined here are all static (or quasi-static). Dynamic phenomena including impacts and vibrational motion are not captured in a static simulation such as this. However, contact elements are allowed to slide against each other (quasi-statically), and to break contact if necessary. Due to the nonlinear nature of the problem and in order to obtain convergence, the solutions are determined using numerical continuation, such that the applied force is increased in small increments until the full applied force is reached. In this finite element analysis, we do not consider any fatigue models. The objective of this work is not to model the damage in fine detail, but instead to study some of the main features (e.g., friction, geometry, boundary conditions) which may create large stresses.

The finite element model constructed here is two-dimensional axisymmetric, which exploits the symmetry of the components of the machine. This is a slight approximation, as the axisymmetry of the 4.45 MN DWM is interrupted by the presence of boltholes, but is justified based on the uniform wear pattern observed on the conical seat joints. Only the portion of the machine where the damage occurred is modeled. This is based on the assumption that the large stress is local to the conical joint.

Galling involves phenomena occurring on different length scales [6]. For instance, galling starts with micron and sub-micron asperities, and can result in millimeter-scale material flow. Here, we assume that the small length scale features will effectively behave according to Coulomb's friction law as $F_{t}=\mu F_{n}$, where $F_{t}$ is the frictional force (tangential force), $\mu$ is the coefficient of friction, and $F_{n}$ is the normal force between the surfaces.

\subsection{Material Constitutive Relations}

All calibrated components of the 4.45 MN DWM are made of stainless steel. The pick-up studs are made of grade AISI 416 (UNS S41600), while the remaining components are made of AISI 410 (UNS S41000). Although these two grades of stainless steel generally have differences in mechanical properties such as hardness, yield strength, and tensile strength, they have essentially the same Young's modulus and Poisson's ratio. Fabrication records of the machine provide only the $0.2 \%$ proof yield strength and the ultimate tensile strength of test specimens of the materials.

We consider two material constitutive relations, linear-elastic and linear-elastic, perfectly plastic (hereafter referred to as elastic-plastic), which are plotted in Fig. 3. This plasticity model requires one plasticity parameter to define the onset of plastic deformation, namely the yield stress $\sigma_{y}$. Although other, more comprehensive plasticity relations may be used, they require additional parameters. We do not have the necessary experimental data of the weight material behavior to determine the additional parameters needed to fully define other plasticity models. However, since the observed plastic deformations are large, we are not concerned with modeling the details of the onset and transitional behavior from the elastic to plastic region, but rather the intent of the elastic-plastic material relation is to predict the conditions where large plastic deformations may occur. From Fig. 3, it is clear that the linear-elastic constitutive relation can result in artificially high stresses, while the plasticity model (elastic-plastic) sets a maximum allowed von Mises stress in the material. In this study we model the behavior of the 4.45 MN DWM using each of the two material constitutive relations. The constitutive relations are described in terms of three parameters,

${ }^{2}$ Certain commercial equipment, instruments, or materials are identified in this article in order to describe the experimental procedure adequately. Such identification is not intended to imply recommendation or endorsement by the National Institute of Standards and Technology, nor is it intended to imply that the materials or equipment identified are necessarily the best available for the purpose. 


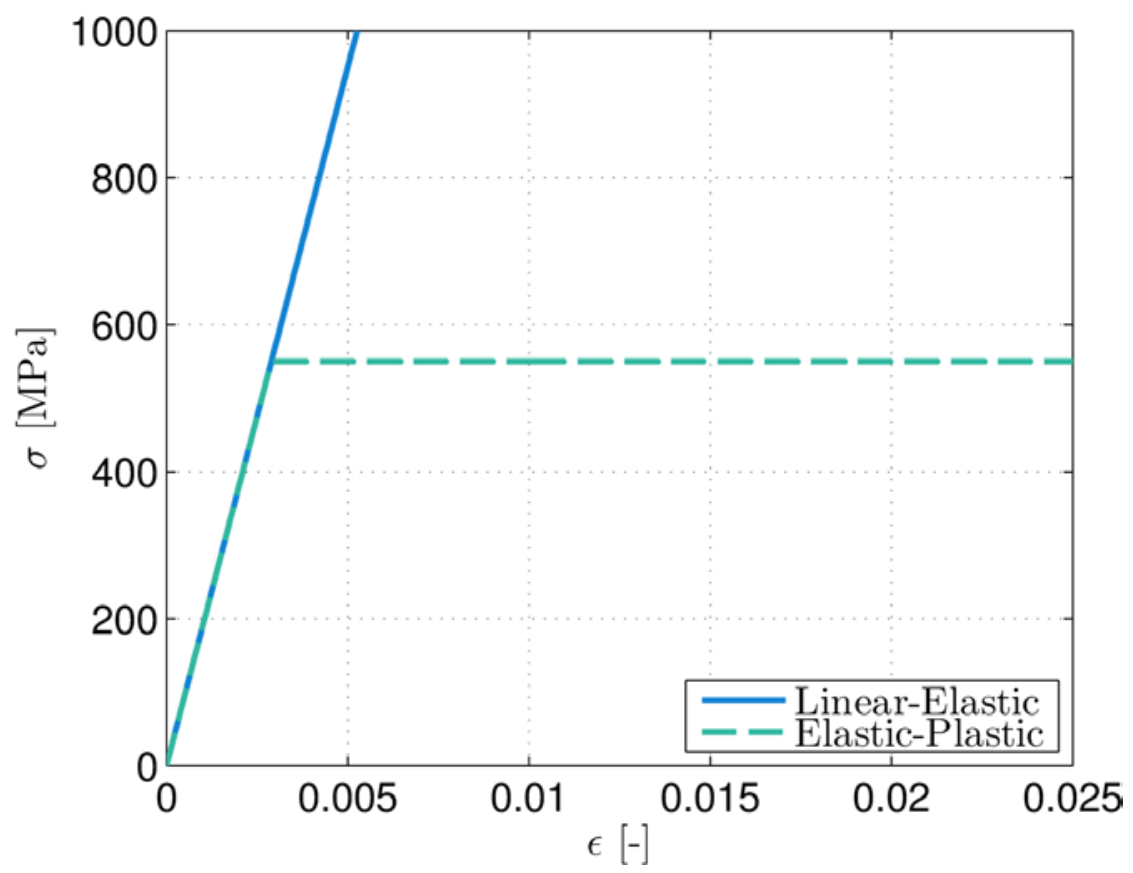

Fig. 3. Stress-strain constitutive relations for two different material laws. Plotted are the two relations used in this study. The relations are shown in terms of axial stresses and strains for the case of a thin rod in tension.

namely the Young's Modulus $E=200 \mathrm{GPa}$, Poisson's ratio $v=0.285$, and the tensile yield stress $\sigma_{y}=$ 550 MPa. Young's Modulus and Poisson's ratio were taken from Ref. [10], while the yield stress was taken to be the $0.2 \%$ yield stress from experimental data taken on test specimens at the time of fabrication of the 4.45 MN DWM weights. The governing equations for the elastic-plastic material laws are given in the COMSOL nonlinear structural mechanics user guide [11].

\subsection{Geometry, Meshing, and Boundary Conditions}

The geometry of a cross-section of the pick-up stud and bottom hub plate assembly is shown in Fig. 4. Since the simulation being performed is axisymmetric, only half of the cross-section is shown. The boundary conditions of the bottom hub plate are set by their clamping to the weight body and the top hub plate, and are not known exactly due to the uncertainty of prestress in the bolts. We consider two separate boundary conditions for the bottom hub plate, which are respectively more-restrictive and less-restrictive of deflection than is the true boundary condition. These are shown in Fig. 4(a). In the first case, the top and side surfaces are fixed (hereafter referred to as the "fully-clamped" case), while in the second case, only the side is fixed and the top is left free.

The mating surfaces of the pick-up stud and bottom hub plate are designated as contact surface pairs in order to be meshed with contact elements. In such a contact pair, one surface is designated as the source and the other as the destination, such that the destination boundaries cannot penetrate the source boundaries [12]. An axial force is applied to the pick-up stud to account for the remaining masses suspended below. The body force due to the gravitational acceleration is also included.

A graphic of the finite-element discretization mesh is provided in Fig. 4(b). The pick-up stud was modelled by combining five sections, while the bottom hub plate was modelled with four sections. This allowed the regions incorporating the contact surfaces on the stud and hub to be meshed with mapped, quadrilateral elements (with mid-nodes), while remaining sections were meshed with triangular elements (with mid-nodes). The quad elements with mid-nodes at the contact surface have a regular geometry which helps to minimize numerical errors. A total of 27000 quadrilateral elements and 148000 triangular 
(a) Geometry and Boundary Conditions:

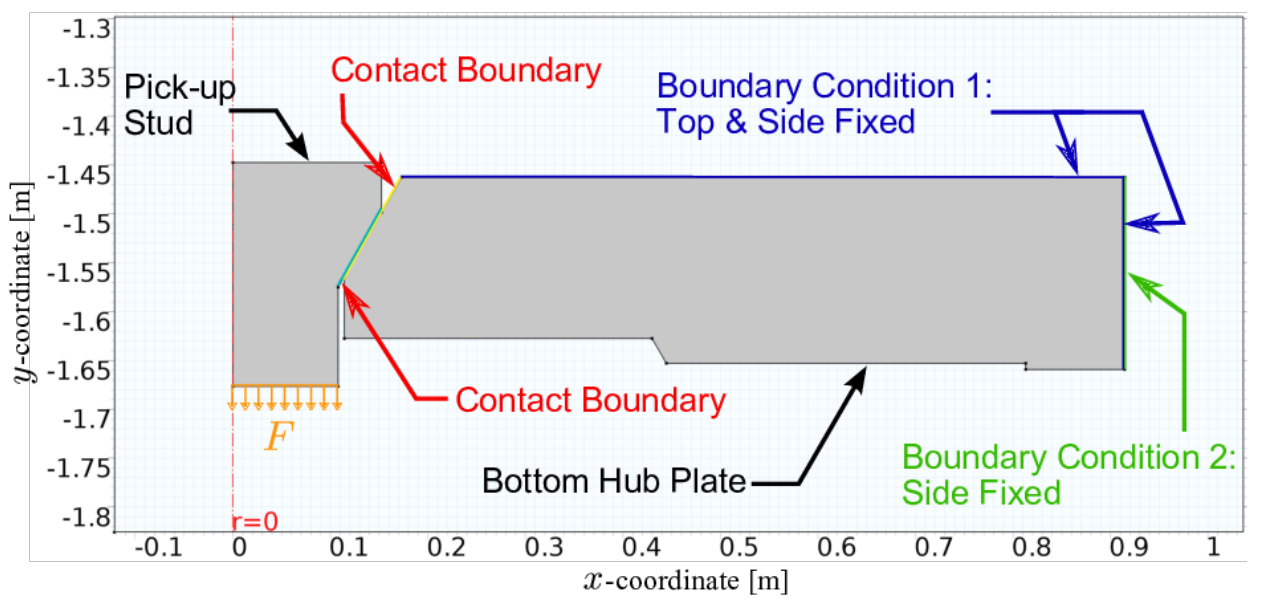

(b) Mesh and Refinement:

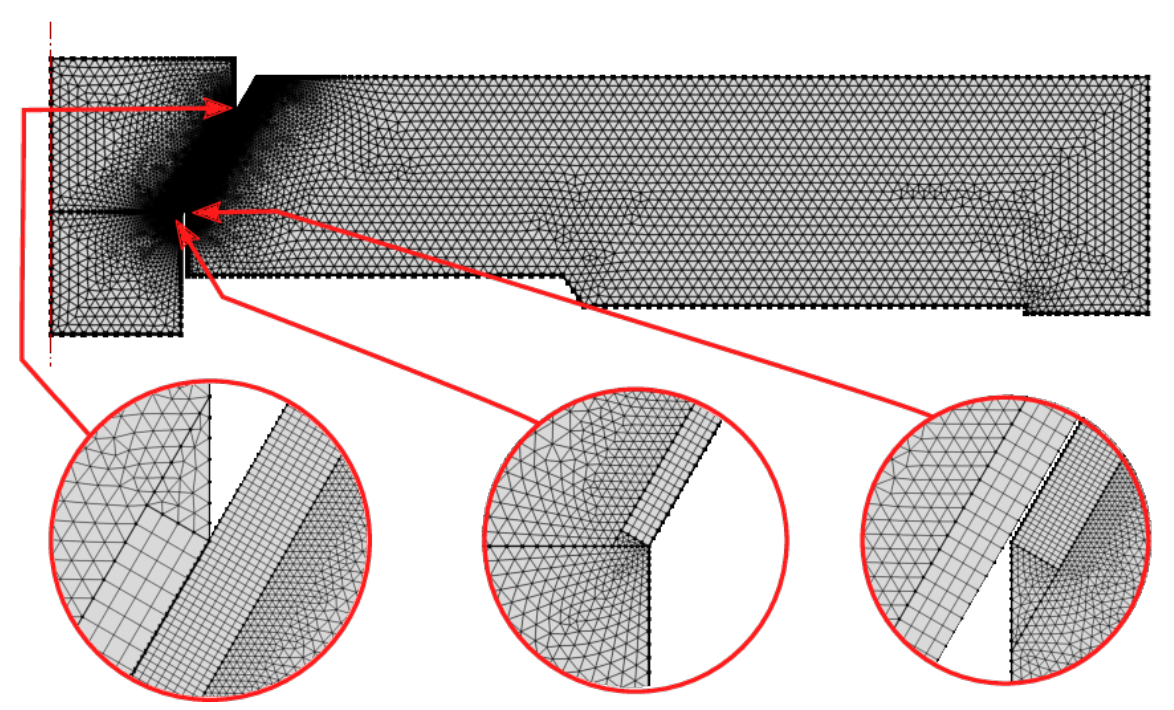

Fig. 4. (a) Geometry, loading, and boundary conditions and (b) finite-element discretization and mesh refinement.

elements were used with an average element quality factor of $0.9708^{3}$. This final mesh was determined after doing a grid and convergence study and is described in detail in Sec. 3.5. The two components of the assembly (the pick-up stud and the bottom hub plate) must be set up to initially have a small penetration of one into another in order to make the starting point of the first iteration unique (i.e., to make the problem well-posed or nonsingular). The initial penetration must be chosen heuristically, as an insufficiently small penetration can lead to the formation of singular matrices, while too large of an initial penetration will cause the problem to be stiff making it difficult to achieve a converged solution. In Fig. 4(b), the hub is taken to be the destination boundary and the stud is taken to be the source boundary. As seen in the expanded sections of Fig. 4, the destination is meshed more finely than the source for numerical stability purposes. For some of the geometric tolerance studies, it was found beneficial to switch the source and destination boundaries.

\footnotetext{
${ }^{3}$ The element quality factor is a dimensionless parameter between 0 and 1 , where 1 corresponds to an element with perfectly regular geometry and 0 represents a degenerate element $[13,14]$. Acceptable meshes generally have element quality factors greater than 0.1 [13].
} 


\subsection{Generating Solutions}

The contact elements, friction, and elastic-plastic material relations are represented by nonlinear equations with a given set of constraints. The solutions are generated using numerical continuation with the applied force $F$, where the total value of $F$ is broken into five equal segments. The nonlinear constraints originating from contact and friction are incorporated into the solution through means of an unconstrained optimization problem. This was implemented using two adjustable penalty parameters, namely one penalty factor for contact (i.e., a surface normal force) and one penalty factor for friction (i.e., a surface tangential force). The default penalty factor values were multiplied by a factor less than unity, typically 0.3 to 0.5 , in order to ensure convergence of the solution, at the cost of computation time. A discussion of penalty factors for contact problems is presented in the software manual [12].

\section{Simulation Results}

\subsection{Initial Results: Boundary Conditions and Material Models}

Simulation results, which are presented in terms of the von Mises stress, for both boundary conditions and a linear-elastic material law are shown in Fig. 5. The von Mises stress was selected to be the indicator of the stress field since it is well suited for predicting failure in the case of static loading of ductile materials in which the tensile and compressive strengths are equal [15]. Here, it is assumed that there is no friction and that the stud dimensions are exact to the original drawings. With the color scale used in Fig. 5, there is no clearly discernible difference between the linear-elastic and elastic-plastic stress fields. The color scales used in plotting the stress fields are saturated at $300 \mathrm{MPa}$ for clarity, but stresses in the structure may be larger than $300 \mathrm{MPa}$. The stress field for the side-fixed boundary condition is shown in Fig. 5(a) and (b), while the fully-clamped boundary condition is shown in Fig. 5(c) and (d). The full threedimensional revolved bodies are shown in Fig. 5(b) and (d). For the side-fixed boundary condition, only the top portion of the stud is in contact with the hub and the bottom portion of the hub and stud do not make contact due to the bending of the hub plate. The stresses shown at the bottom of the hub plate originate from hoop stresses in the circular body, and not from contact with the stud. In the fully-clamped case, the highest stresses occur in the bottom portion of the stud where it makes contact with the hub. We note that this location is where galling was observed in the components of the machine. The remaining contour plots will show the stress distribution in a two-dimensional cross-section of the hub and stud, as the simulation is axi-symmetric.

The von Mises stress on the bottom hub plate, expressed as a function of position along the contact arc, is shown in Fig. 6 for both boundary conditions and material relations. The stresses plotted here are the stresses at Gauss points located at a depth of $1 \mathrm{~mm}$ below the surface (equivalently eight elements in depth) at the interface between the quadrilateral and triangular elements, since the stresses at the surface can have numerical errors resulting from the residual in the solution of the nonlinear optimization problem. In the fully-clamped boundary condition of Fig. 6, high stresses are produced in the bottom area of the hub contact surface (between $0 \mathrm{~cm}$ to $1 \mathrm{~cm}$ ) because the stud and hub are in contact. In the side-fixed case there are also stresses (smaller in magnitude), but these are hoop stresses due to the deformation of the hub. In the region of $7 \mathrm{~cm}$ to $8 \mathrm{~cm}$, the top of the stud makes contact with the hub and all four scenarios produce high stresses. Here, the linear-elastic material relation results in von Mises stresses up to $800 \mathrm{MPa}$, but the elastic-plastic material relation allows for plastic deformations such that the stresses are capped at 550 MPa. 
(a) Side-Fixed Boundary Condition

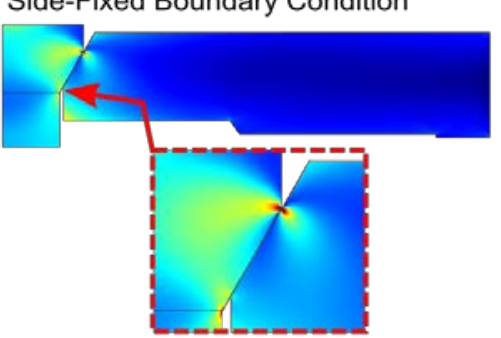

(c) Fully-Clamped Boundary Condition

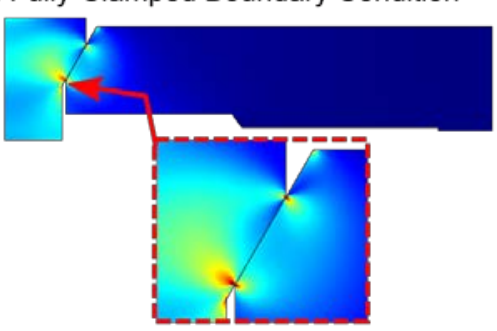

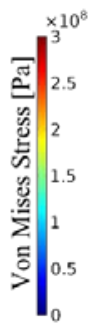

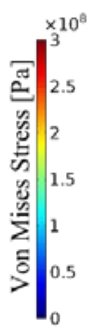

(b) Revolved: Side-Fixed Boundary Condition
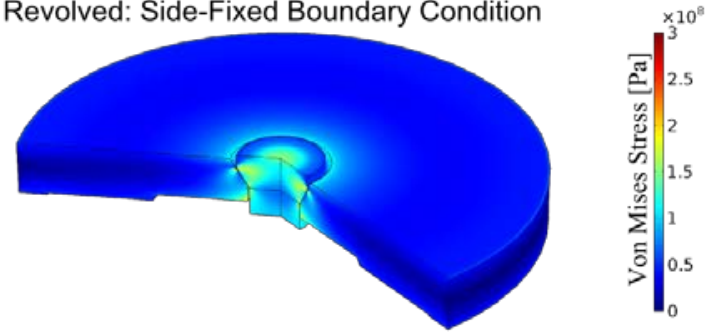

(d) Revolved: Fully-Clamped Boundary Condition

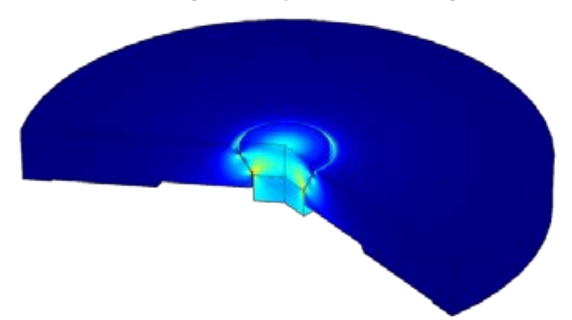

Fig. 5. Simulation results for a linear-elastic material relation: (a) side-fixed boundary condition, (b) 3D revolution of the side-fixed boundary condition, (c) fully-clamped (top-and-side-fixed) boundary condition, and (d) 3D revolution of the fully-clamped boundary condition.

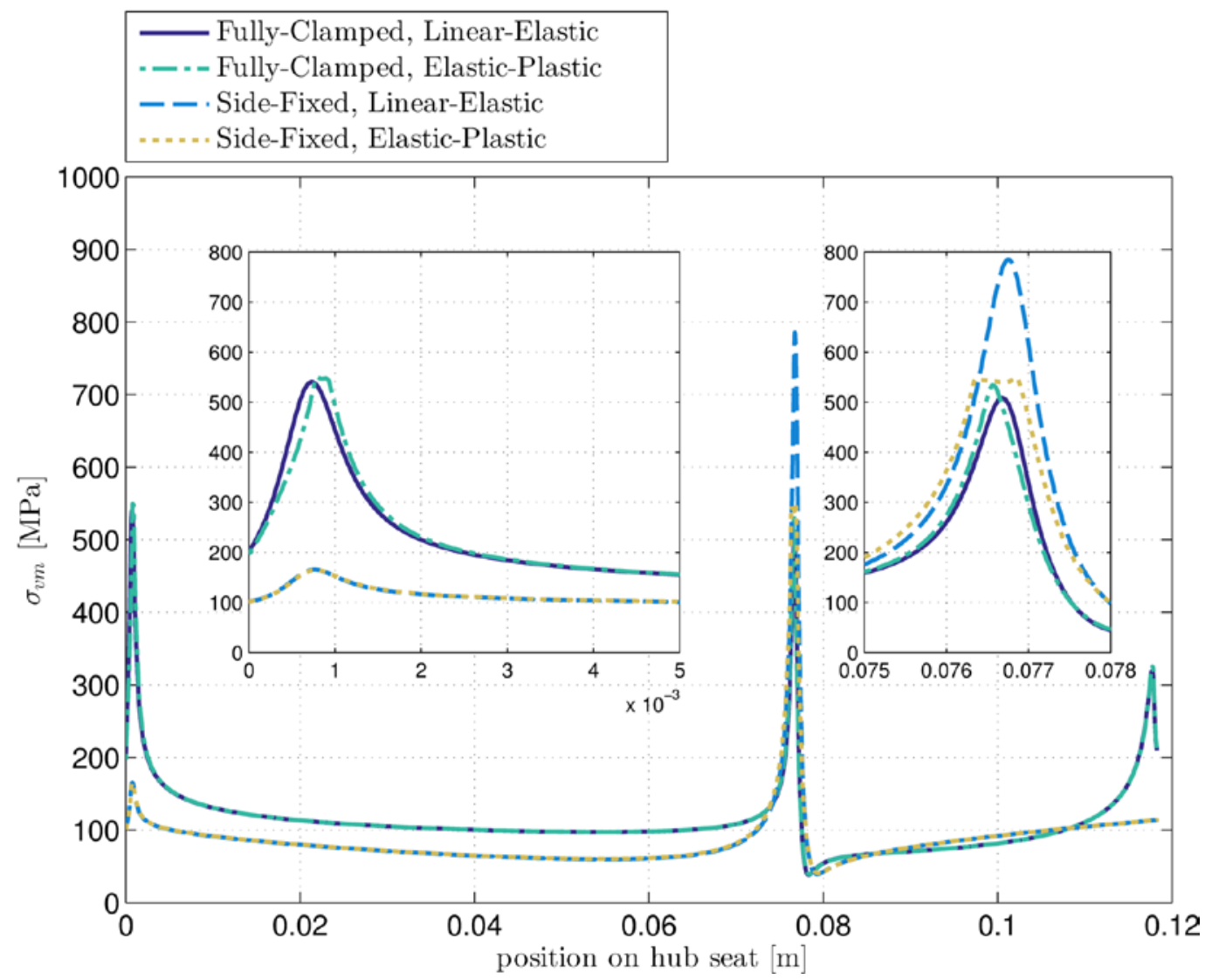

Fig. 6. Von Mises stress over the hub contact surface for the different material relations and boundary conditions. Position 0 corresponds to the lowest point (i.e., minimum diameter point) on the contact arc. 


\subsection{Loading Effects}

The fully-clamped boundary condition with the plastic material relation is shown in Fig. 7 for five different load values. From the shape evolution of the force profile, as well as the magnitude of peak stress, the resultant von Mises stress is in general nonlinear with respect to the applied force. We observe that the location of the peak stress near the top of the stud (at approximately $7.7 \mathrm{~cm}$ ) moves toward the base of the stud as the force is increased. Although data are not provided, during the simulated loading of the structure tangential relative motion (i.e., sliding) between the surfaces of the stud and hub was observed, which is a key ingredient for surface wear.

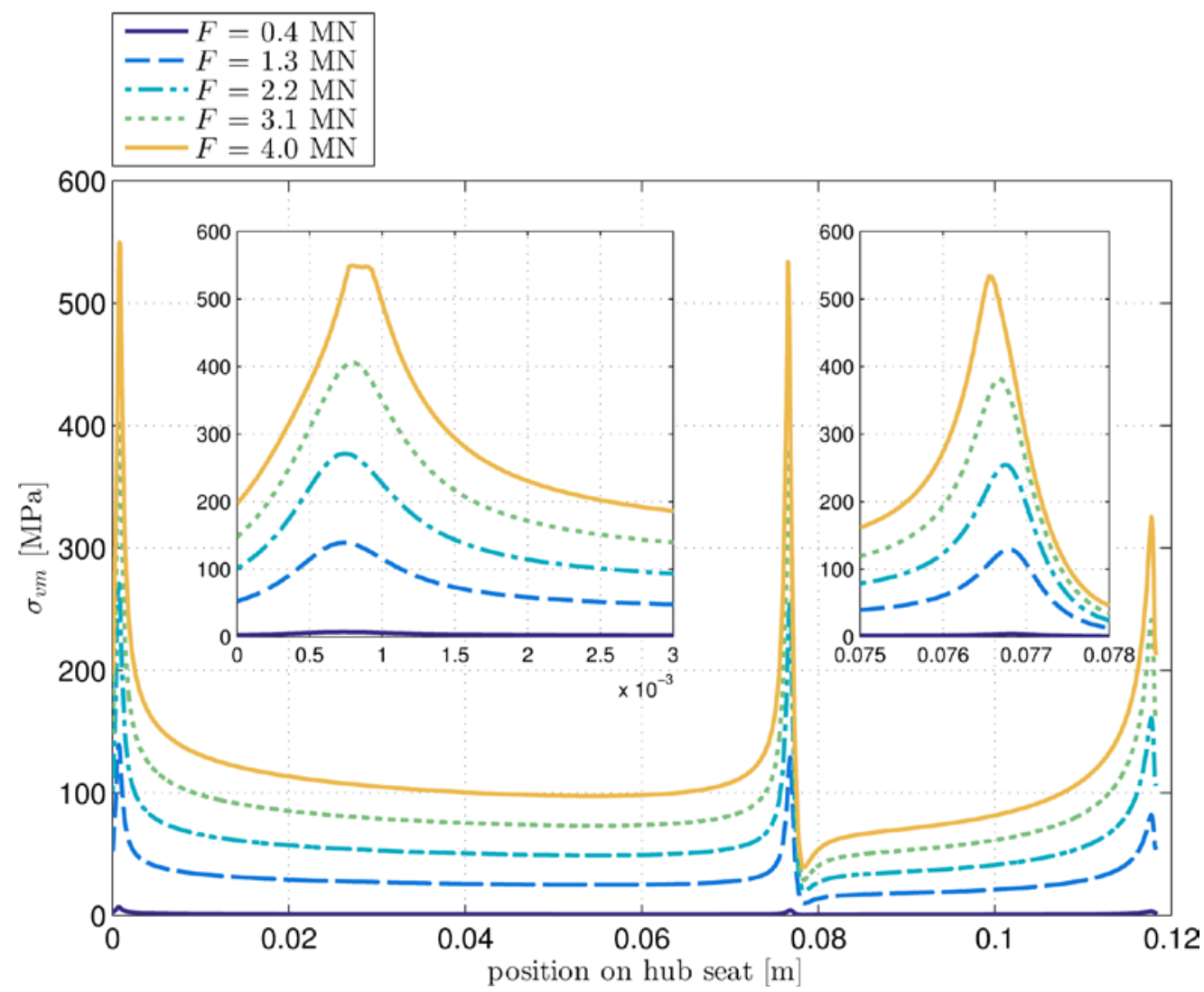

Fig. 7. Von Mises stress over the hub contact surface for the fully-clamped boundary condition and the elastic-plastic material relation while varying the applied load.

\subsection{Frictional Effects}

In this section, we study the effects of the coefficient of friction between the contact surfaces of the stud and hub. The stress field of the simulated structure is shown in Fig. 8 for five different values of $\mu$. Additionally, the von Mises stress along the conical seat of the hub plate is given in Fig. 9 for the fullyclamped boundary conditions with a plastic material relation. The results shown in Figs. 8 and 9 are at the maximum load of 4.0 MN. Perhaps not intuitively, the higher levels of friction actually decrease the peak levels of von Mises stress, while increasing the value of the minimum von Mises stress. These results are interpreted as follows. When the coefficient of friction between the two surfaces is zero, only the vertical component of the normal force supports the stud. Thus, the normal force must be large due to the angle of the two contact surfaces. Furthermore, there is zero external tangential force at the interface in this zero friction case. These together result in higher von Mises stresses. On the other hand, when the coefficient of friction is in a moderate range of values $(\mu>0.1)$, both the normal force and friction (a tangential force) 


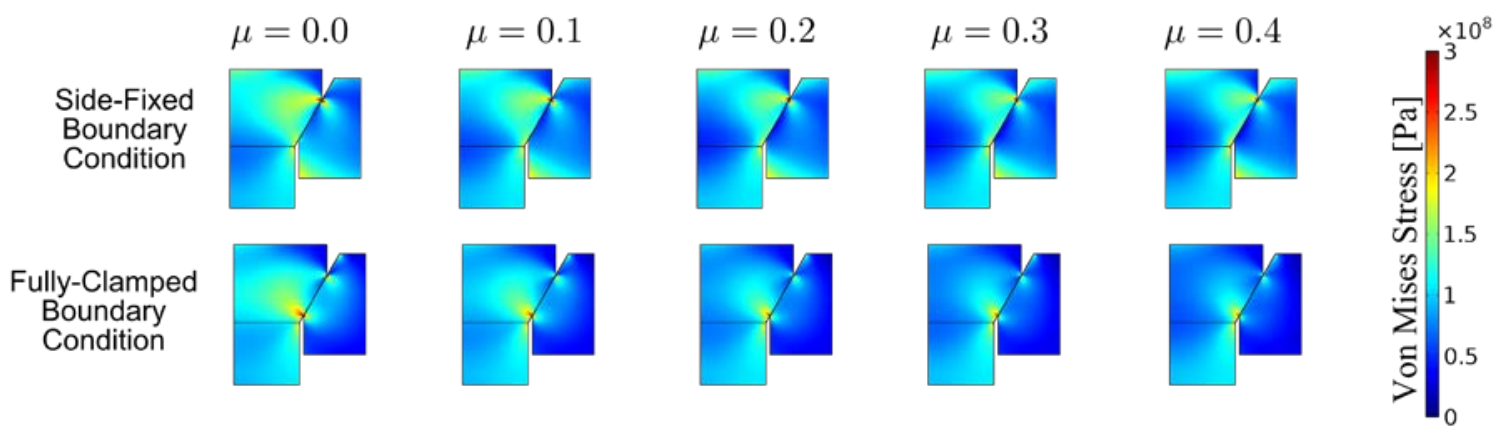

Fig. 8. Evolution of the stress field for two different boundary conditions and different values of friction at maximum load.

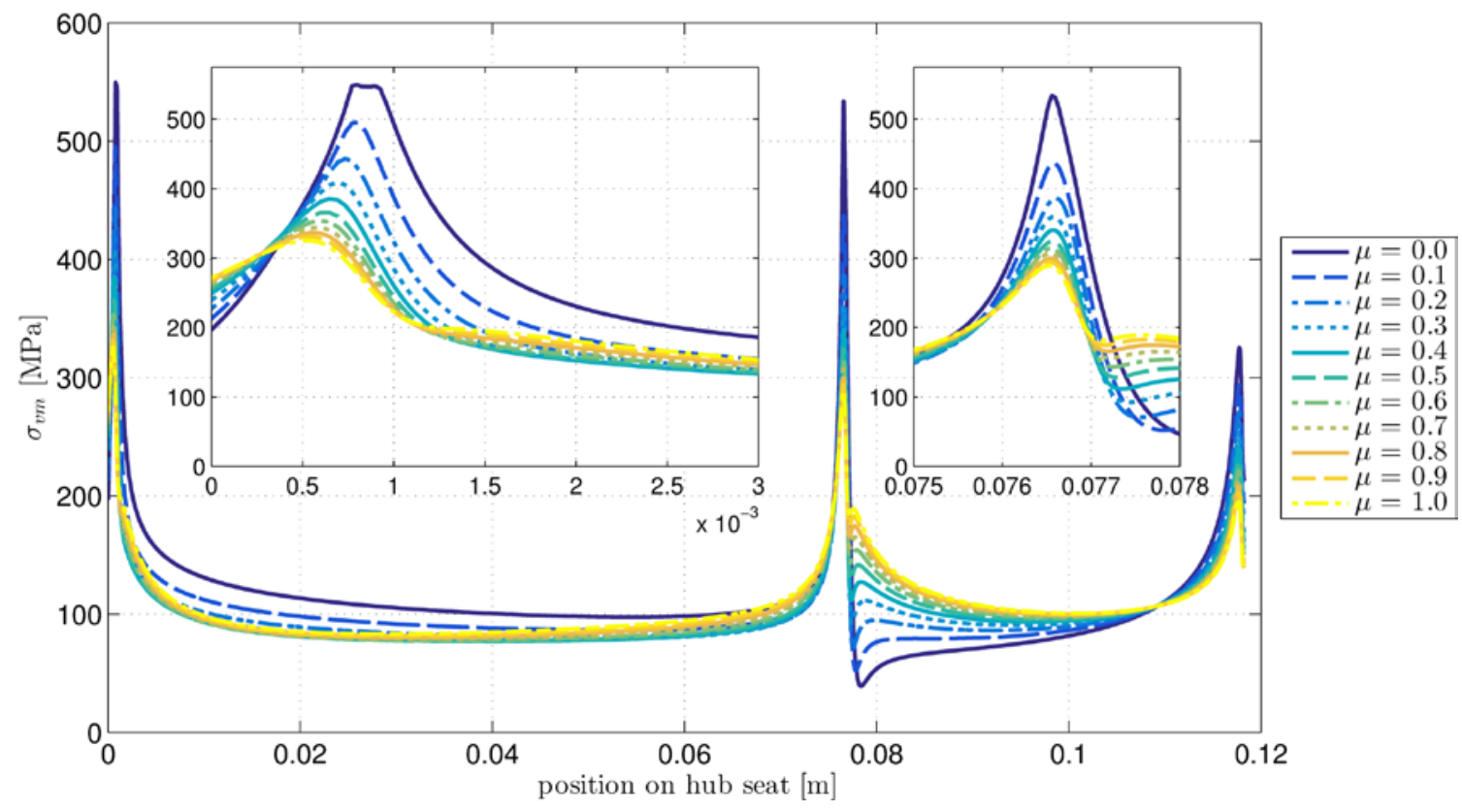

Fig. 9. Von Mises stress over the hub contact surface for the fully-clamped boundary conditions and the elastic-plastic material law while varying the coefficient of friction $\mu$.

have a vertical component that balance the downward force component exerted by the stud, and the resultant von Mises stresses are smaller.

Although the higher levels of friction can be beneficial in lowering the peak von Mises stresses, high frictional forces may be detrimental from a tribological standpoint, as the mechanisms by which such a larger frictional force would be generated (such as larger quantities and magnitudes of asperities) may increase the tendency of the two surfaces to gall. Therefore, from a tribological perspective, inserting an intermediate material (i.e., a surface coating or lubricant) to prevent the initial adhesion of the two surfaces may be preferable [7]. We note that the 1.33 MN DWM that showed similar damage in the early 1970s and was treated with a lubricant, namely $\mathrm{MoS}_{2}$ solid or semi-solid, has not experienced a reoccurrence of such damage up to the present. Additionally, the $498 \mathrm{kN}$ DWM that was restored in 1988 was treated with a $\mathrm{MoS}_{2}$ solid lubricant. 


\subsection{Dimensions and Tolerances}

The relative angle of contact between the pick-up stud and the bottom hub plays a critical role in the resulting stresses throughout the surface region. The angle of the pick-up stud and bottom hub are designed to meet at an angle of $30^{\circ} \pm 0.25^{\circ}$ from the center axis of the stud and hub. The hub plates and the studs were measured using a set of digital calipers, and the angle (with respect to the center axis) of the contact surface on each of the top three studs and weight bodies was determined to be within the tolerance specified on the fabrication drawing, namely $30^{\circ} \pm 0.25^{\circ}$. Dimensions of the hub seats were not measured before remachining the damaged surfaces. The measured angles for the studs are listed in Table 1. Each stud angle was calculated from measurements of three linear dimensions. These measurements were performed at three separate locations around the circumference and averaged. The uncertainty in Table 1 corresponds to two standard deviations of the measurements. It is observed that the first pick-up stud in the stack, which is screwed into the weight body No. 3 , has an angle that is closer to $30^{\circ}$ than the next two studs which showed damage. This may be the reason that the first stud did not show any damage, while the following two in the stack experienced galling.

Table 1. Summary of the stud angular measurements as found (before remachining). Asterisks (*) denote studs with significant damage. The uncertainties represent twice the standard deviation of three measurements of the angle.

\begin{tabular}{cc}
\hline Stud Weight No. & Angle \\
\hline $5-3$ & $29.98^{\circ} \pm 0.025^{\circ}$ \\
$5-4^{*}$ & $29.89^{\circ} \pm 0.049^{\circ}$ \\
$5-5^{*}$ & $29.90^{\circ} \pm 0.041^{\circ}$ \\
\hline
\end{tabular}

A parametric study was performed in order to determine the effects of the contact surface angles on the stress in the conical seat. The nominal angle of $30^{\circ}$ on the bottom hub was varied by an amount $\alpha$ that ranged from $-0.05^{\circ}$ to $0.05^{\circ}$ for the fully-clamped boundary condition. This boundary condition was selected because the stress field calculated in this case for the nominal contact angle value (see Figs. 5 and 6) agreed qualitatively with the damage pattern observed in the machine (i.e., the maximum stresses predicted in the FEA were located near the smaller diameters of the conical seat). Additionally, the elasticplastic material law was used in these simulations (see Fig. 7). The $0.01^{\circ}$ gradations correspond to diameter gradations of approximately 0.001 inches, a typical machining tolerance increment on analog lathes. It is noted that the measured hub angle errors are larger than the maximum simulated angular mismatch by a factor of approximately two, so that the stress fields actually occurring in the machine are expected to be higher than those observed in this parametric study. Von Mises stress fields within the stud and bottom hub are shown in Fig. 10, while the stress along the hub seat is shown in Fig. 11. These figures were generated at the maximum load and with a friction coefficient of 0.1 , but the parametric study results for the geometry are largely independent of the coefficient of friction.

Although the angular variation $\alpha$ is small, the stress field changes drastically within the stud and the hub. From Fig. 11, the optimum angular deviation (with zero applied force) for the fully-clamped boundary conditions is $0.00^{\circ}<\alpha_{\mathrm{opt}}<0.02^{\circ}$. This optimum angle physically represents a small initial clearance at the bottom of the hub and stud, and initial contact near the top. Upon loading, the stud deforms in a way to match the surface of the hub. Additionally, when two surfaces are in contact initially at the top (as opposed to the bottom) of the conical joint, there is a larger initial area of contact (due to the larger diameter) resulting in smaller stresses. It is noted that if the other boundary conditions were selected (side-fixed, topfree), the indicated optimum angle would likely be a negative value of $\alpha$ (corresponding to an initial gap near the top), since the bottom hub plate would bend to close the top gap. From the simulation results of the different geometric angles, even in the presence of deflections of the bottom hub plate, $\alpha=0.00^{\circ}$ is still reasonably close to the optimum angle. Additionally, with the angle $\alpha=0.00^{\circ}$, as the bottom hub plate flexes down, the stud and hub remain in contact at the larger diameter of the stud, so the stresses are lower than if they were in contact at the bottom portion of the stud. 

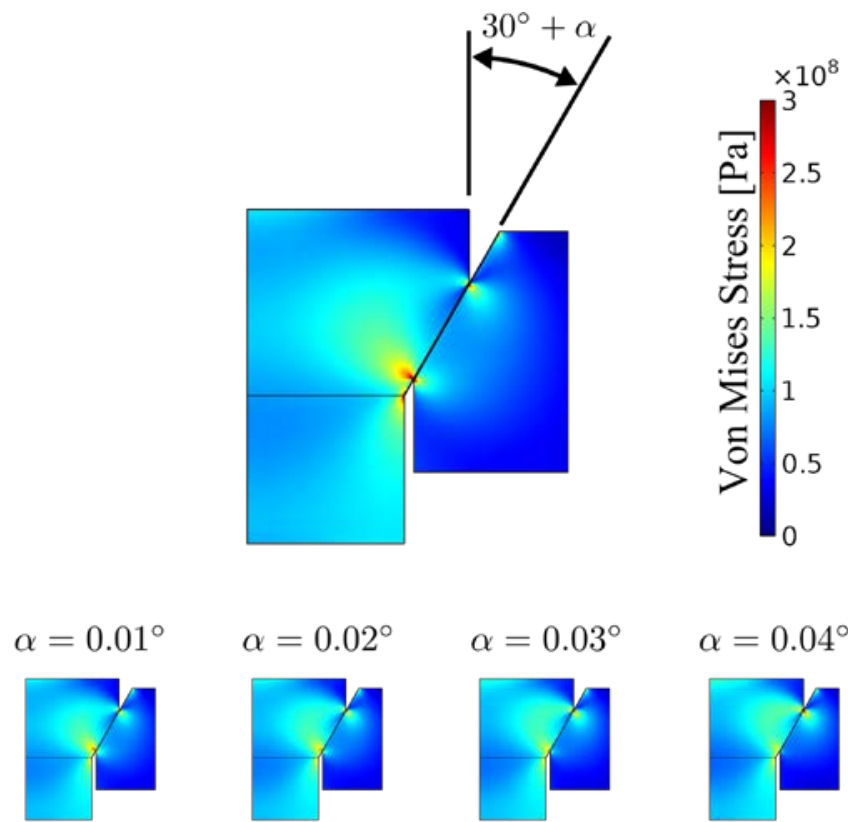

$\alpha=0.02^{\circ}$

$\alpha=0.03^{\circ}$

$\alpha=0.04^{\circ}$

$\alpha=0.05^{\circ}$
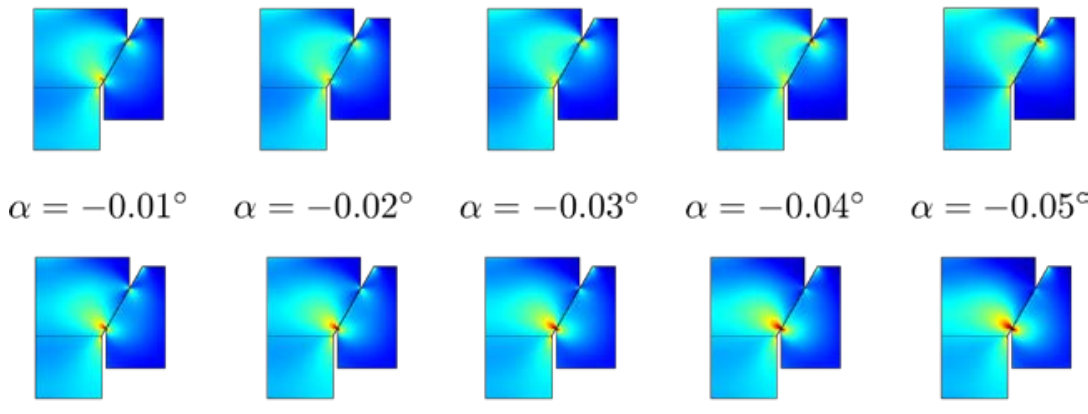

$\alpha=-0.03^{\circ}$

$\alpha=-0.04^{\circ}$

$\alpha=-0.05^{\mathrm{c}}$

Fig. 10. Von Mises stress over the hub contact surface for the fully-clamped boundary condition and the elastic-plastic material law while varying the angle $\alpha$ between the two mating surfaces.

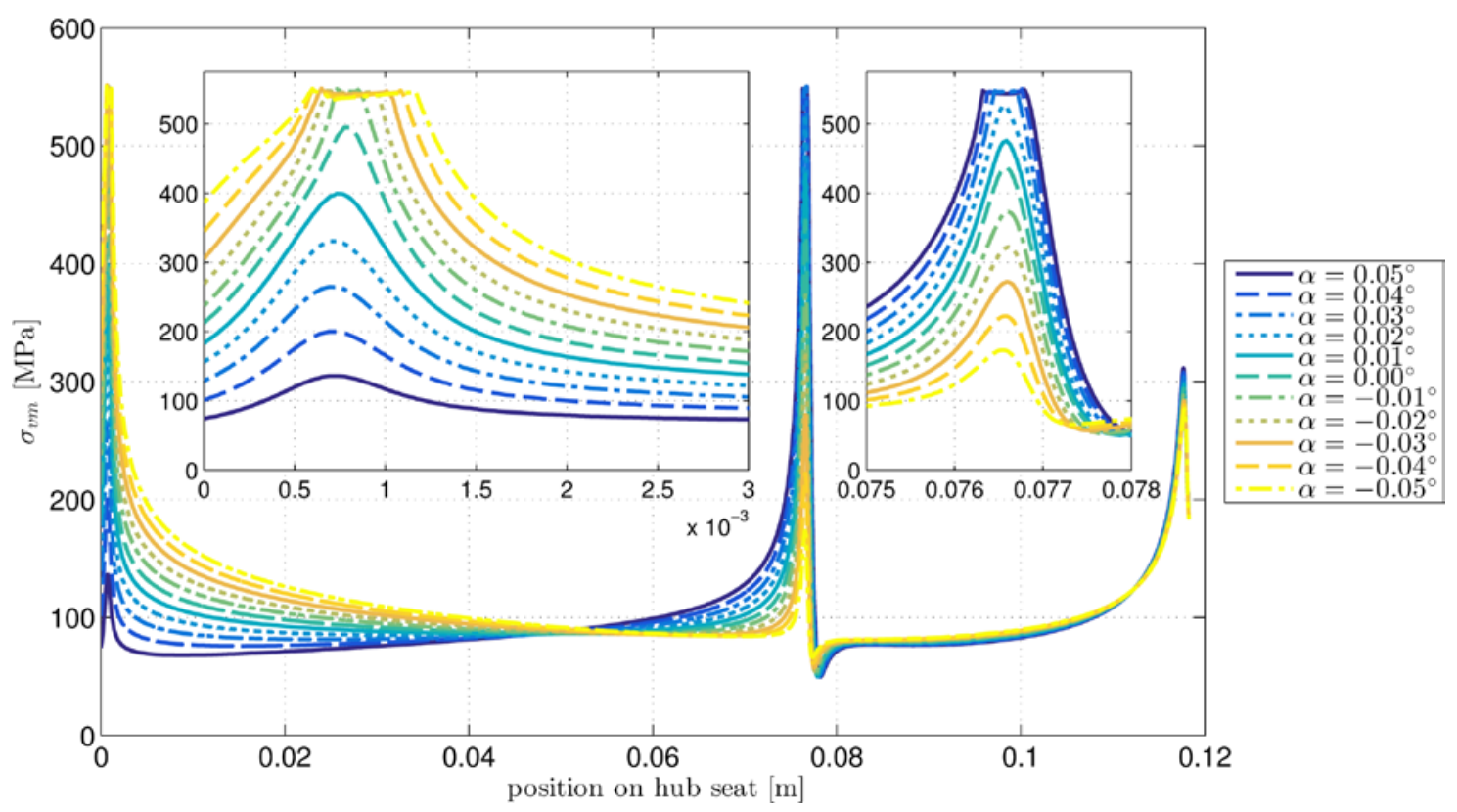

Fig. 11. Von Mises stress over the hub contact surface for the fully-clamped boundary condition and the elastic-plastic material relation while varying the angle $\alpha$ between the two mating surfaces. 


\subsection{Analysis of Error}

Here we report the analysis that was used to determine the accuracy of the modeling of the physics and the accuracy of the simulation results. Specifically, the implemented checks were:

(i) The stress field in the cylindrical portion of the stud agreed with the stress in a uniaxial rod under simple tension to better than $1 \%$.

(ii) The reaction force at the boundary was equal to the sum of the applied forces and the gravitational body forces to within $1 \%$. This is an important check when solving nonlinear problems.

(iii) The number of elements used in the contact region was doubled until such doubling did not significantly change the calculated stresses at the interface. The value of the stress change at the final doubling step was less than $1 \%$ change in von Mises stress for over $99 \%$ of the elements in the contact region.

\section{Conclusions}

The NIST deadweight machines that are presently used to realize force were conceived in the late 1950's and were constructed in the early-to-mid 1960's, without the benefit of computerized finite-element analysis. These machines have realized force standards to a high degree of accuracy for several decades. Here, we present a modern finite element analysis of the contact joints in the NIST deadweight machines, specifically for the largest such DWM - the 4.45 MN DWM - in which damage occurred at some of these joints. There are a few conclusions which can be drawn from this study:

(i) The top-and-side-fixed (fully-clamped) boundary condition, which physically models strong clamping in the bolts that hold the weight bodies together, accurately predicts the location of damage observed in the machine.

(ii) The analysis suggests that plastic deformations at the contact surfaces likely began with first operation of the machine at its full capacity.

(iii) With the benefit of hindsight and a finite-element simulation (results not shown in this manuscript), it is found that a larger contact diameter would have been beneficial by reducing the contact stresses at the pick-up joints in the upper part of the DWM.

(iv) Small contact angle differences (even within the specified fabrication tolerances) result in drastically different stress fields and resultant stress magnitudes throughout the structure.

(v) Although the top three pick-up studs had dimensions within their specified tolerances, the first pick-up stud in the stack had an angle that was closer to the specified $30^{\circ}$ than the other two studs. This is one possible reason why damage did not occur at the first contact joint, but occurred at the next two.

(vi) Higher levels of friction can lower the von Mises stresses at the contacting surfaces, but may have an overall tribological effect that is detrimental.

(vii) Precisely determining an optimum angular mismatch between the contact surfaces is difficult given the uncertainty in the boundary conditions; however, a zero relative angle between the two surfaces or a slight initial opening at the bottom of the stud, so that contact occurs at the top, gives the smallest resultant stresses throughout the structure.

At the time of writing, the current state of the DWM and the plans for completing the repair are as follows. The pick-up studs and the bottom hub seats on the damaged pieces have been remachined to their specified drawings and measured with a coordinate measuring machine. The contact surfaces of the pick-up stud and bottom hub have been treated with a $\mathrm{MoS}_{2}$ solid lubricant to mitigate seizure of the two pieces. After recalibration of the masses, the machine will be reassembled. For the initial break-in of the DWM, the weights will be loaded in a manner to promote elastic shake-down or work-hardening of the contact joints. This will be done by loading and unloading the machine cyclically working up in $222 \mathrm{kN}$ increments until the entire weight stack is lifted. For example, the loading sequence will take the form $\{0 \mathrm{kN}, 222 \mathrm{kN}$, $0 \mathrm{kN}\},\{0 \mathrm{kN}, 222 \mathrm{kN}, 444 \mathrm{kN}, 222 \mathrm{kN}, 0 \mathrm{kN}\}$, etc. In addition to the work hardening, the break-in sequence may also cause plastic deformations of the surfaces to minimize the difference in relative angle (corresponding to $\alpha=0$ ) at least locally on the surface, which will aid in minimizing stresses. 


\section{References}

[1] Jabbour ZJ, Yaniv SL (2001) The kilogram and measurements of mass and force. J Res Natl Inst Stan 106(1):25-46. http://dx.doi.org/10.6028/jres.106.003

[2] Bartel T (2005) Uncertainty in NIST force measurements. J Res Natl Inst Stan 110(6):589-603. http://dx.doi.org/10.6028/jres.110.084

[3] Bartel T, Yaniv S, and Seifarth R (1997) Force measurement services at NIST: equipment, procedures, and uncertainty. Proc 1997 Natl Conf of Stand Lab Workshop and Symposium 1:421-431.

[4] Yaniv SL, Sawla A, Peters M (1991) Summary of the intercomparison of the force standard machines of the National-Instituteof-Standards-and-Technology, USA, and the Physikalisch-Technische-Bundesanstalt, Germany. J Res Natl Inst Stan 96(5):529540. http://dx.doi.org/10.6028/jres.096.029

[5] Bartel T (2012) Final report on force key comparison CCM. F-K4. a and CCM. F-K4. b for 4 MN and 2 MN forces. Metrologia 49(1A):07003.

[6] Williams JA (1994) Engineering tribology (Cambridge University Press, New York).

[7] Shpenkov GP (1995) Friction surface phenomena (Elsevier Science B.V., Amsterdam, The Netherlands).

[8] Hassan T, Kyriakides S (1992) Ratcheting in cyclic plasticity, part I: Uniaxial behavior. Int J Plast 8(1):91-116. http://dx.doi.org/10.1016/0749-6419(92)90040-J

[9] Hassan T, Corona E, Kyriakides S (1992) Ratcheting in cyclic plasticity, part II: Multiaxial behavior. Int J Plast 8(2):117-146. http://dx.doi.org/10.1016/0749-6419(92)90010-A

[10] Henry SD, Moosbrugger C, Anton GJ, Sanders BR, Hrivnak N, Terman C, Kinson J, Muldoon K, Scott WW (2001) ASM handbook (ASM International, Materials Park, $\mathrm{OH}$ ).

[11] COMSOL (2013) Nonlinear mechanics module user's guide (COMSOL, North America), Version 4.3b.

[12] COMSOL (2013) Structural mechanics module user's guide (COMSOL, North America), Version 4.3b.

[13] COMSOL (2012) COMSOL multiphysics user's guide (COMSOL, North America), Version 4.3b.

[14] Hughes TJR (2000) The finite element method: linear static and dynamic finite element analysis (Dover Publications, Mineola, New York).

[15] Norton RL (2006) Machine design: an integrated approach (Pearson Prentice Hall, Upper Saddle River, New Jersey).

About the authors: Nicholas Vlajic is a staff scientist currently working in the area of dynamic force calibration. Ako Chijioke is a staff scientist, and principal investigator for the dynamic force calibration project. Rick Seifarth has been with NIST over 33 years, and is the principal investigator of the large force calibration group. The National Institute of Standards and Technology is an agency of the U.S. Department of Commerce. 